\title{
Optical Evaluation of an As-Manufactured Compound Secondary Concentrator
}

\author{
Donald A. Jaworske ${ }^{1}$, Timothy J. Skowronski², and Barry J. Miles ${ }^{3}$ \\ 'NASA Lewis Research Center, 21000 Brookpark Rd., Cleveland, OH 44135 \\ ${ }^{2}$ Cleveland State University, Cleveland, OH 44121 \\ ${ }^{3}$ BWX Technologies, Inc. P.O. Box 785, Lynchburg, VA 24505
}

\begin{abstract}
Secondary concentrators are needed in solar thermal propuision to further concentrate the energy collected by large lightweight primary concentrators. Although the physics of secondary concentrators has been worked out in detail and the manufacturing has been successfully completed for a ground demonstration, there is a need to quantify the specific performance of as-manufactured concentrators. This paper summarizes the properties of a secondary concentrator manufactured for the Integrated Solar Upper Stage engine ground demonstration in 1997 and presents data obtained from the optic that describe the performance of the as-manufactured component.
\end{abstract}

\section{INTRODUCTION}

Solar thermal propulsion is being considered as a means of transferring payloads from low Earth orbit to other orbits of interest. In this concept, one or more large lightweight mirrors, or primary concentrators, are used to collect and focus the sun's energy into the aperture of one or more heat receivers. Heat from these receivers is used to heat a gas for propulsion. The concept requires sunlight to be concentrated by a factor of 8000 to 1 . To achieve this concentration ratio, a primary concentrator with a concentration ratio of 4000 to 1 is optically coupled to a secondary concentrator with a concentration ratio of 2 to 1 .

One type of secondary concentrator, a compound parabolic concentrator (CPC), has been used successfully in a ground demonstration of a solar thermal propulsion system (Westerman and Miles, 1998). This CPC was designed for high temperature operation and utilizes the high temperature materials graphite, rhenium, and iridium in its construction. Although the theoretical performance of the CPC has been worked out in detail (Welford and Winston, 1978), an evaluation of the performance of the as-manufactured CPC was needed to understand the overall efficiency of the optic, as part of understanding the overall thermal system.

The objectives of this paper are to describe the system used for evaluating the as-manufactured compound secondary concentrator and to present in graphical form the results of the optical measurements. A discussion of potential improvements follows.

\section{MATERIALS AND METHODS}

The CPC used in this study was one of three designed and built by BWX Technologies for use in the Integrated Solar Upper Stage program. This one was a spare. The optic, manufactured from Poco graphite, was first shaped to the desired contour. A coating of rhenium was deposited onto the graphite and lapped to remove major surface imperfections. A coating of iridium was deposited onto the rhenium and polished to a mirror-like finish to provide the reflective surface. The fidelity of the contour and the reflectivity of the surface define the performance of the optic. 
To accomplish the goal of evaluating the performance of the as-manufactured CPC, a chopped laser beam was used to illuminate the optic from a variety of defined entrance angles. A detector was then used to capture the reflected light from a variety of defined exit angles. The position of the incoming laser beam was either rotated about, or translated away from, a defined entrance angle origin.

For the rotational study, the optic was mounted horizontally on an optics bench such that the centerline of the optic was parallel to the surface of the optics bench. An entrance angle origin was defined by dropping a perpendicular across the entrance plane, through the centerline of the optic. The laser beam was directed through the entrance angle origin, as shown in Figure 1, and a protractor mounted on the optics bench at the base of the perpendicular was used to read the entrance angles with respect to the laser source.

For the translation position study, the optic was moved horizontally on the optics bench such that the laser beam at a given entrance angle was translated along an entrance plane radius. Translation positions at a given entrance angle between $0^{\circ}$ and $40^{\circ}$ were recorded in $6.35 \mathrm{~mm}(0.25 \mathrm{inch})$ increments, with the first increment beginning when the laser beam engaged the optic at the rim of the exit plane.

The origin for all exit angles was defined by dropping a perpendicular from the exit plane, through the centerline of the optic. A protractor was mounted on the optics bench at the base of this perpendicular to read the exit angles with respect to the detector.

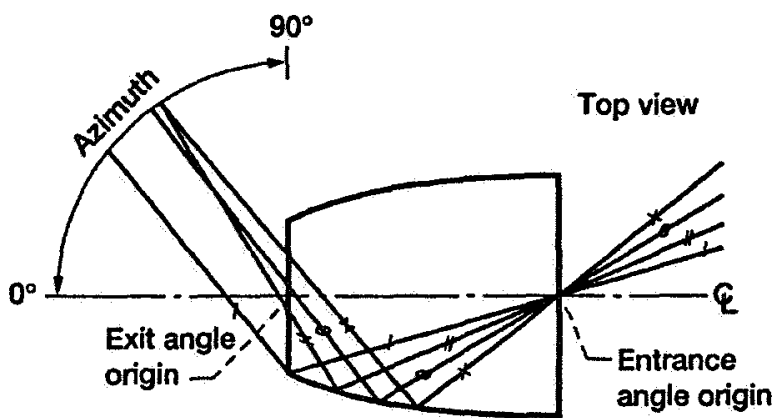

FIGURE 1. Schematic diagram of the optic, shown in cross section, with entrance origin and exit origin defined.

The laser, centerline of the optic, and detector were all set to the same height above the optics bench. The detector height could be set to seven different non-overlapping positions, centered on the centerline of the optic. In this way, it was possible to position the detector at a variety of azimuth and elevation values, with respect to the exit plane origin. By obtaining intensity as a function of azimuth and elevation, for each entrance angle and translated position, it was possible to generate a 3-D graph of the intensity output surrounding the optic. Intensity input was obtained by swinging the detector into the path of the laser, in front of the optic.

The purpose of using a chopped laser beam was to remove the effect of any drift in the laser. This was accomplished by passing the laser beam through a chopper and a half silvered mirror, sending half of the beam to the optic and the other half to an integrating sphere equipped with a reference detector identical to the one adjacent to the optic. Optic detector values were normalized to the reference detector values according to Equation (1):

$$
I_{\text {cor, } t}=I_{\text {ref, o }} \times\left(I_{s, i}\left(I_{\text {ref, }}\right)\right.
$$

where $I_{\text {cor, } t}$ is the corrected intensity recorded in $\mathrm{mV}$ at time $t, I_{\text {ref, o }}$ is the reference intensity in $\mathrm{mV}$ initially, $I_{s, t}$ is the sample intensity in $\mathrm{mV}$ at time $\mathrm{t}$, and $\mathrm{I}_{\text {ref, }}$ is the reference intensity in $\mathrm{mV}$ at time $\mathrm{t}$. Although intensity was recorded in $\mathrm{mV}$ by the detector, it is understood that intensity is power per unit area. A linear response of the detector in $\mathrm{mV}$ corresponds to a linear response in intensity. This normalization procedure was carried out for all intensity values and it was found that the laser actually drifted very little during a test, typically less than $0.6 \%$.

Finally, a Perkin-Elmer Lambda-9 spectrophotometer equipped with a $15 \mathrm{~cm}$ diameter integrating sphere was used to evaluate the reflectivity of an iridium coated witness coupon with respect to angle. This was accomplished by 
utilizing an attachment to the integrating sphere that allowed the witness coupon to be mounted in the center of the sphere at various angles. Reflectivity values at $632 \mathrm{~nm}$ were obtained at $5^{\circ}$ increments.

\section{RESULTS AND DISCUSSION}

Figure 1 showed the approximate contour used to manufacture the optic. The optic was designed for an acceptance angle of $34.5^{\circ}$. Visual inspection of the optic revealed good adherence of the coatings used in the manufacturing process. The outermost iridium layer appeared reflective to the unaided eye. However, it was difficult to obtain a specular image with the eye.

Using the entrance angle and exit angle geometry defined above for the rotational angle study, Figure $2 a-j$ summarizes the output of the optic, as expressed in three dimensional plots of exit intensity versus azimuth and elevation. The $30^{\circ}$ entrance angle had the greatest intensity value, $550 \mathrm{mV}$. The input to the optic, as measured by intercepting the laser beam in front of the optic, was $4240 \mathrm{mV}$.

It is interesting to note that the maximum exit intensity occurs between $55^{\circ}$ and $65^{\circ}$ regardless of entrance angle, when using the defined entrance and exit origins. It is also interesting to note that the base of the three dimensional plots shown in Figure 2 give some indication of the degree of spreading that occurs as the laser beam leaves the optic. These observations suggest that the optic, when illuminated by a primary concentrator, will tend to generate an annulus of intensity at an exit angle of $55^{\circ}$ to $65^{\circ}$. Such an annulus is consistent with the design, and in a graphite receiver this "hot spot" should be dissipated around the cavity within a few reflections.

An attempt was made to "sum" the area under one of the curves in Figure 2 to compare the total integrated intensity at the exit with the intensity impinging on the optic at the entrance. This approach should have provided a numerical value between zero and one, a value indicative of the "efficiency" of optical throughput. This exercise was not successful, perhaps owing to the uncertainties brought about through the summation process. An alternative approach is discussed later.

The geometry of the optic and the observed exit angles were used to identify the angle of incidence at several points tangential to the surface of the optic. Four such tangential points are shown in Figure 1. These angles of incidence were used as target values in the Lambda-9 integrating sphere study to evaluate the reflectivity of an iridium witness coupon as a function of angle in the vicinity of these target values.

Using the scheme defined above for the translation position study, exit intensity versus azimuth and elevation was obtained as the laser beam entered the optic from several points along a radius of the entrance aperture. These data were collected at several entrance angles by first setting the entrance angle of interest at the entrance plane origin and then by translating the optic to different positions along the radius. Figure $3 \mathrm{a}-\mathrm{g}$ summarizes a translation position study starting with an entrance angle of $20^{\circ}$. As one moves the laser beam along the radius, the peak intensity of the three dimensional plots moves from approximately $65^{\circ}$ with the laser beam just engaging the optic at the exit aperture rim, to $35^{\circ}$ with the laser beam at the $25.4 \mathrm{~mm}$ translation position. At translation positions greater than $25.4 \mathrm{~mm}$, the intensity at the exit dropped off significantly suggesting that the laser beam was being reflected back out the entrance aperture. This is consistent with CPC theory. Again, the three dimensional plots shown in Figure 3 give some indication of the degree of spreading that occurs as the laser beam leaves the optic. As before, the input to the optic was measured by intercepting the laser beam in front of the optic, and was $4320 \mathrm{mV}$. Other entrance angles between $0^{\circ}$ and $40^{\circ}$ showed similar trends.

From the translation position study one can infer that the light entering the optic from a primary concentrator at angles less than the acceptance angle and crossing the entrance plane at other than the entrance plane origin will exit the optic through the exit plane at angles less than the $55^{\circ}$ to $65^{\circ}$ "hot spot" annulus. In other words, when the optic is mated to the graphite receiver this light will be directed further back into the graphite receiver. Light impinging on the optic somewhere near the front edge will be lost because of reflection back through the entrance of the optic. 

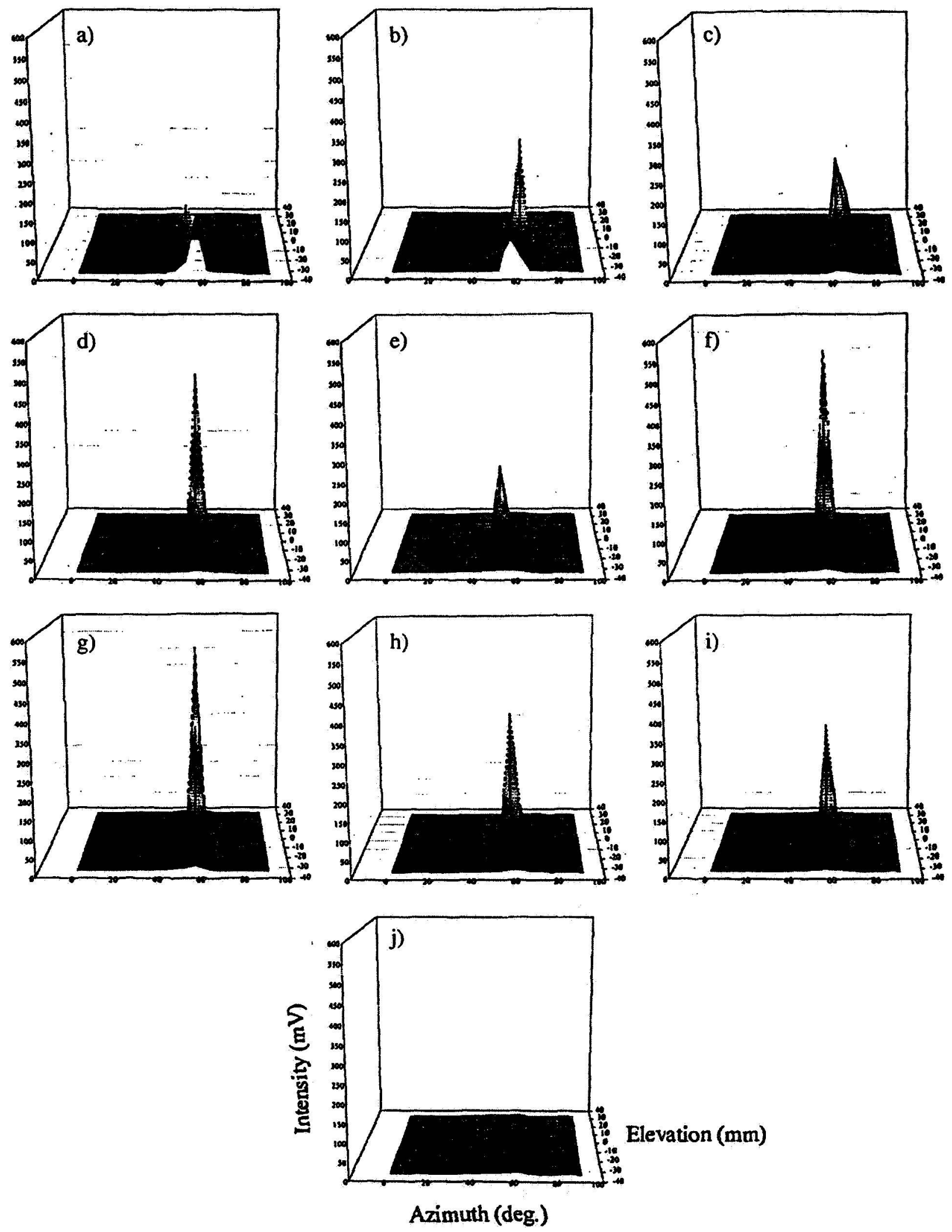

FIGURE 2. Exit intensity as a function of azimuth and elevation for entrance angles of a) $17.5^{\circ}$, b) $20^{\circ}$, c) $22.5^{\circ}$, d) $25^{\circ}$, e) $27.5^{\circ}$, f) $30^{\circ}$, g) $32.5^{\circ}$, h) $35^{\circ}$, i) $37.5^{\circ}$, and j) $40^{\circ}$. 

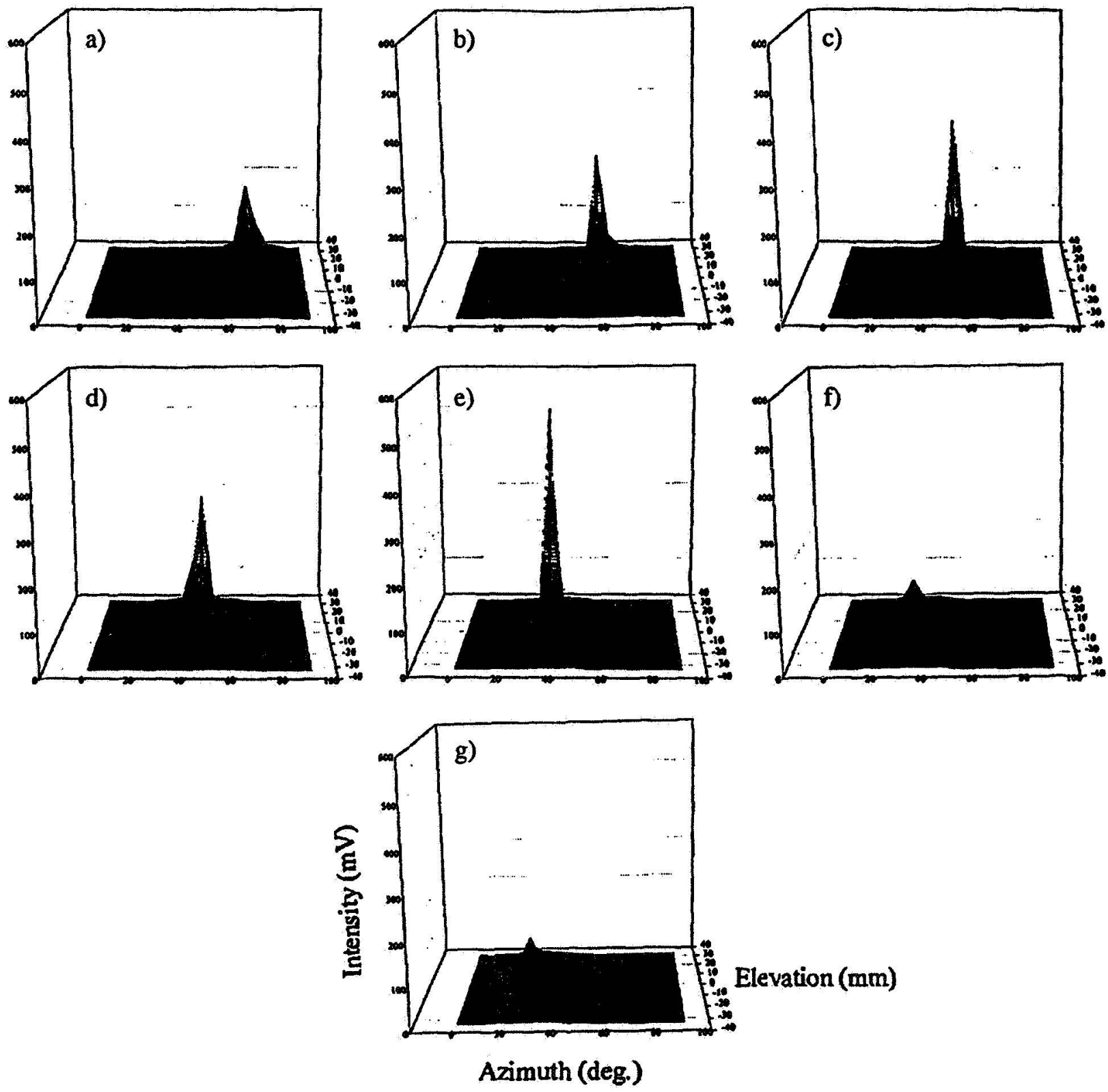

FIGURE 3. Exit intensity as a function of azimuth and elevation for an entrance angle of $20^{\circ}$ and translation positions of a) 0, b) $6.3 \mathrm{~mm}$, c) $12.7 \mathrm{~mm}$, d) $19.05 \mathrm{~mm}$, e) $25.4 \mathrm{~mm}$, f) $31.75 \mathrm{~mm}$, and g) $38.10 \mathrm{~mm}$.

Finally, the Lambda-9 evaluation of the reflectivity of an iridium witness coupon at $632 \mathrm{~nm}$ as a function of angle is summarized in Figure 4. In general, the reflectivity remains nearly constant out to $50^{\circ}$, and gradually drops off thereafter. As indicated previously, many of the reflections in the optic occur at an angle of incidence in the vicinity of $46^{\circ}$ to $56^{\circ}$. Hence, the efficiency of the optic should not be modeled by a single, near normal, reflectivity value. 


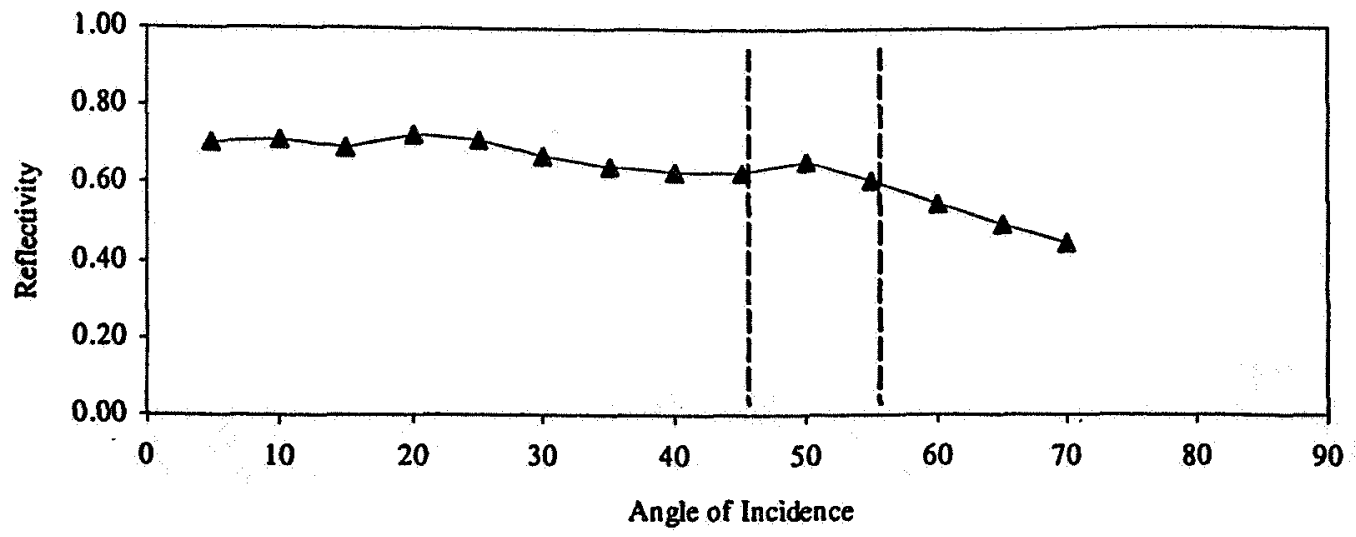

FIGURE 4. Reflectivity, at $632 \mathrm{~nm}$, of an iridium witness coupon as a function of angle of incidence. The region between $46^{\circ}$ and $56^{\circ}$ indicates the reflectivity expected for the compound parabolic concentrator studied here.

\section{FUTURE WORK}

One idea for improving the technique to measure optical throughput is to utilize an integrating sphere at the exit of the optic. In this way, light from the exit is captured in its entirety. The integrating sphere would accept light from the optic after a laser beam has engaged the optic. It could also accept light from the laser without engaging the optic, by allowing the laser beam to pass through the optic on the center line. The ratio of these two values would yield the optical throughput, or efficiency, of the optic. Ideally, one would like to use a converging light source analogous to a primary concentrator to illuminate a sector of the optic, or perhaps even the entire optic.

\section{CONCLUSIONS}

A chopped laser beam arrangement was used to identify the performance of an as-manufactured compound parabolic concentrator. Laser light directed onto the optic from a variety of entrance angles primarily exited the optic at between $55^{\circ}$ and $65^{\circ}$. Three dimensional plots of the output of the optic, plotted as intensity versus azimuth and elevation, revealed that there was some minor spreading of the beam. The implication is that light collected by the optic from a primary concentrator would be distributed in an annulus inside the receiver. Laser light directed onto the optic from a variety of positions translated along a radius exited the optic at lesser angles. Light directed onto the optic near the front edge was reflected back out the front and lost. Evaluation of the reflectivity of an iridium witness coupon measured as a function of angle suggests that a single, near normal, reflectivity value should not be used to model the efficiency of the optic. Rather, an integrating sphere coupled to the optic ought to provide a means to measure optical efficiency.

\section{REFERENCES}

Welford, W. T., and Winston, R., The Optics of Nonimaging Concentrators, Academic Press, New York, pp. 1-200, 1978. Westerman, K. O., and Miles, B. J., "Manufacture and Test of a Bimodal Solar Power and Propulsion Receiver," Proceedings of the $33^{\text {rd }}$ Intersociety Energy Conversion Engineering Conference, IECEC-98-310, 1998. 